\title{
Performance Evaluation and Delay Modelling of VolP Traffic over 802.11 Wireless Mesh Network
}

\author{
Amit Chhabra \\ Dept. of CSE \\ SDDIET, Barwala \\ Panchkula, India
}

\author{
Dr. Gurpal Singh \\ Dept. of CSE \& IT \\ BBSBEC, Fategarh Sahib \\ Punjab, India
}

\begin{abstract}
This paper analyzes voice quality in terms of R-Factor and MOS in IEEE 802.11s Wireless Mesh Network (WMN). Another issue addressed in this paper is the effect of increasing nodes on voice transmission in wireless mesh network. The simulation model developed allows identifying the main reasons for voice quality degradation in Mesh network. Results show that voice quality measured in terms of R-Factor and MOS degrades with increase in number of hops (Wireless Mesh Points) in Wireless Mesh network. Wireless mesh network has been modeled using M/D/1 queue and based on this analytical delay model, the average one way delay suffered by Voice over IP (VoIP) traffic has been calculated. Simulations have been conducted to validate the correctness of the analytical model.
\end{abstract}

\section{General Terms}

Wireless Mesh Network, VoIP, Delay, Jitter, Packet Loss, Codecs

\section{Keywords}

Wireless Mesh Network, Voice over IP (VoIP), R-Factor, Mean Opinion Score (MOS), H.323, G.711

\section{INTRODUCTION}

Wireless Mesh Networks have recently emerged as an important Research area worldwide. A WMN consists of static mesh routers and mesh clients [1]. Mesh routers or Mesh Points have minimal mobility i.e. they work without any energy constraints and form the mesh backbone for mesh clients. However, the mesh clients can be either stationary or mobile node. Wireless mesh networks are an attractive communication paradigm because of their low cost and relative ease of deployment [2]. The capability of being self-organized, auto-configurable and self-healing makes the wireless mesh networks a very reliable and robust choice [2],[3]. WMNs typically consist of many base stations, some of which are directly connected to the Internet. The users connect to one of the base stations, and the base stations form a multi-hop wireless network to route traffic between the Internet and the users. Figure 1 shows the basic architecture of WMN where all mesh points or routers are connected with each other. The WMN backbone provides alternative paths between each pair of endpoints, increasing communications reliability and eliminating single points of failure within the mesh [4]. Mesh Point Portals with the gateway/bridge functionalities for mesh network connects it to the Internet as well as with other networks.

In this paper, a delay model for WMN has been developed and subsequent simulations have been conducted to analyse the performance of VoIP. The major performance metrics including
Average Delay, R-Factor and Mean Opinion Score (MOS) for VoIP have been evaluated by varying number of mesh points in Wireless mesh network. In this work, M/D/1 queue based model has been developed and delay incurred by the VOIP traffic has been calculated.

This paper is organized into 8 Sections. Section II discusses fundamentals of VoIP and Section III contains brief introduction to Voice Quality Metrics like Delay, Packet Loss, Jitter, R-Factor and MOS. Section IV describes the related work done in this area. Section V describes the Analytical Delay Modeling of the WMN and section VI presents the simulation results. Section VII presents comparison between analytical and simulation results. Finally, Section VIII presents conclusion and remarks regarding the future work.

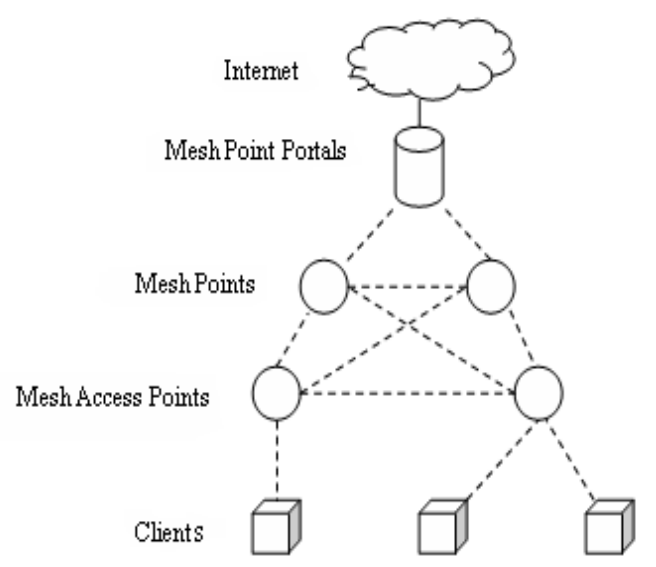

Fig.1 Architecture of WMN

\section{VOIP BASICS}

Voice over Internet Protocol (VoIP) [6] is a technology that transports voice data packets across packet switched networks using the Internet Protocol. VoIP involves digitization of voice streams and transmitting the digital voice as packets over conventional IP-based packet networks like the Internet, Local Area Network (LAN) or wireless LAN (WLAN) [5],[6].Although the quality of VoIP does not yet match the quality of a circuitswitched telephone network. In WLAN, as VoIP technology is still in the early stages of commercial deployment, it is necessary to examine if VoIP over WLAN can provide a Quality of Service (QoS) comparable to that of the existing PSTN and cellular networks. Also as the 802.11 based WMNs is gaining popularity, the research efforts are required to investigate the Quality of Service of VoIP over such multi-hop networks. Figure 2 shows the basic VOIP architecture. 
In a typical VoIP application, a voice signal is sampled, digitized, and encoded using a given algorithm/coder. The encoded data is packetized and transmitted using RTP/UDP/IP [7]. At the receiver's side, data is de-packetized and forwarded to a jitter buffer, which smoothes out the delay incurred in the network. Finally, the data is decoded and the voice signal is reconstructed. In a VoIP system, the total mouth-to-ear delay is composed of three components: codec delay, jitter delay, and network delay. However, WMNs are multi-hop in nature and add additional delay when VoIP traffic is passed over them. Also the delay added by WMN depends on the number of wireless hops over which the traffic has travelled.

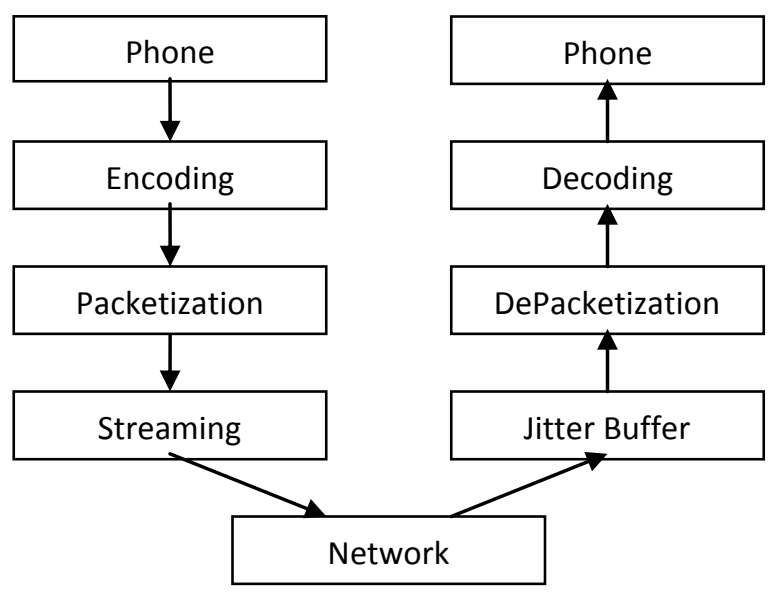

Fig.2. VOIP Architecture

\section{VOICE QUALITY METRICS IN WIRELESS MESH NETWORK}

VoIP has become a killer application and is gradually being tested over emerging areas like Wireless mesh networks. There are various challenges for VoIP in WMN. To date some efforts have been carried out to investigate the performance of VoIP over Wireless Mesh Network [11],[12],[13],[14], and there are many challenging issues which still remains to be resolved.

There are many factors that affect the quality of voice over Wireless mesh network. The quality of a VoIP call [10] is impacted by several parameters such as delay, delay jitter, packet loss and so on. These parameters are determined by the performance of codecs, echo control, buffering and type of network [6],[7].

Delay is the time taken by the VoIP packet to travel from mobile node to gateway in a WMN. Delay can be measured in either oneway or round-trip delay. A typical VoIP traffic can tolerates up to $150 \mathrm{~ms}$ end to end delay in a single direction before the quality of the call becomes unacceptable.

Jitter is the variation in packet measured over time. If the delay of transmissions varies too widely in a VoIP call, the call quality is greatly degraded. The amount of jitter tolerable on the network is affected by the depth of the jitter or playout buffer on the network equipment in the voice path. The more jitter buffer available, the more the network can reduce the effects of jitter. Most VoIP endpoint devices have jitter buffers to compensate for network jitter. The acceptable level of jitter in a network should be less than $2 \mathrm{~ms}$.

Packet loss is losing packets along the data path, which severely degrades the voice application. Voice is not tolerant of packet loss. Even $1 \%$ packet loss can "significantly degrade" a VoIP call. Packet loss can occur because of several reasons: a packet can be discarded in a router because of buffer overflow or because the arriving packet is corrupted, the packet can be accidentally misrouted or be lost because of a link failure.

In this, research work investigates the performance of VoIP traffic as dependent on number of mesh nodes in a multi-hop 802.11based WMN.

\subsection{R-Factor and Mean Opinion Score}

The most reliable method for evaluating the voice quality is the Emodel[8][9] defined by the International Telecommunication Union-Telecommunication (ITU-T). An ETSI work group developed the E-Model also known as European telecommunications Standards Institute (ETSI) Computation Model. The E-Model defines an analytical model for prediction of VoIP quality based on various network impairment parameters such as packet loss, delay, codec type, noise etc.

E-Model gives a single, overall measure of conversational voice quality called the rating factor (R-Factor) [9].R Factor includes the effect of mouth-to ear delay and losses in packet-switched networks and is given by the following relation

$$
R=R_{0}-I_{s}-I_{d}-I_{e}+A
$$

Where $R_{0}$ is voice quality without distortion. Usually it is equal to $100, I_{s}$ impairments caused due to echo and packet loss,$I_{d}$ corresponds to impairment level caused by delay and delay jitter, $I_{e}$ represents the impairment caused by encoding. A is expectation factor that a user can tolerate.

An R-factor between 50 and 60, 60 and 70, 70 and 80, 80 and 90, or 90 and 100 indicates poor, low, medium, high, or best voice quality, respectively. Different approaches have been used to translate these ratings into an overall single measure from which speech quality can be judged. A popular approach is to calculate the arithmetic mean of scores known as a mean opinion score (MOS). A MOS ranges from a minimum of 1 to a maximum of 5 . A MOS of 4.0 or higher is considered toll quality, and a lower limit of 3.0 is suggested for usable telephony. R and MOS are related as follows

$$
M O S=1+0.035 R+7 * 10^{-6} R(R-60)(100-R)
$$

A buffer in the receiving device always compensates for jitter (delay variation). If the delay variation exceeds the size of the jitter buffer, there will be buffer overruns at the receiving end, causing packet loss to occur.

The VoIP QoS in WMN partly also depends on the types of voice codec used [6],[7]. The primary function of a voice codec is to perform analog/digital voice signal conversion and digital compression. There are many codecs available for digitizing speech. Table 1 gives some of the characteristics of a few standard codecs. Among three commonly used codec [8] in Internet telephony are G.711, G.723.1 and G.729. These codecs differ in their coding rate (bps), frame rate (frames/s), algorithmic latency 
that influences the speech quality or Mean Opinion Source (MOS) in a VoIP network. Payload size for each codec depends on the codec speed or data rate. The G.711 has speed of $64 \mathrm{Kbps}$ and if each speech packet size is of $20 \mathrm{~ms}$, then the payload size for G.711 will be of 160 bytes.

$$
\begin{aligned}
& \text { Payload Size }(\text { bytes })=[\text { codec speed }(\text { bits } / \mathrm{sec}) * \\
& \text { speech packet size }(m s)] /[8(\text { bits } / \text { byte }) * 1000 \\
& (m s / \mathrm{sec})]
\end{aligned}
$$

Payload size of 160 bytes for G.711 codec means that the codec produces 160 bytes chunks of VoIP traffic every $20 \mathrm{~ms}$ interval. The G.711 codec gives the best voice quality, since it performs no compression, introduces the least delay, and is less sensitive than other codecs to packet loss. Other codecs, like G.729 and the G.723 consume less bandwidth by compressing the signal. In this research work, G.711 codec has been used because of its good voice quality and least delay.

Table 1. Different Attributes of Codecs

\begin{tabular}{|c|c|c|}
\hline Codec & $\begin{array}{c}\text { Data Rate } \\
\text { (kbps) }\end{array}$ & $\begin{array}{l}\text { Speech Packet } \\
\text { Size (ms) }\end{array}$ \\
\hline G.711 & 64.0 & $20 \mathrm{~ms}$ \\
\hline G.729 & 8.0 & $20 \mathrm{~ms}$ \\
\hline G.723m & 6.3 & $30 \mathrm{~ms}$ \\
\hline G.723a & 5.3 & $30 \mathrm{~ms}$ \\
\hline
\end{tabular}

\subsection{Call Signaling Protocols}

There are several VoIP call signaling protocols [7] like H.323, SIP, MGCP, and megaco/H.248, which can be used for establishment and management of VoIP traffic in WMN. The signaling refers to establishing a connection or an association between a pair of participants in VoIP call. Signaling protocols must also perform address translation, bandwidth management, authorization, and in some cases make routing decisions. H.323 and SIP are peer-to-peer control-signaling protocols, while MGCP and Megaco are master-slave control-signaling protocols.H.323 and Megaco are designed to accommodate video conferencing as well as basic telephony, but they are still based on a connectionoriented paradigm, despite their use for packet communications systems. The Voice-over-IP Activity Group of the International Multimedia telecommunications Consortium (IMTC) recommends H.323, which had been developed for multimedia communications over packet data networks. These packet networks might include LANs or WANs. H.323 was originally developed for videoconferencing over a packet based network, but was quickly adopted for Voice over IP.The main function of the protocol is to perform call control and management on an IP network. In this research work we have used H.323 as a signaling protocol for VoIP in wireless mesh network.

\section{RELATED WORK}

VoIP over WMN has witnessed significant research activity in the recent past. Literature review reveals considerable research work conducted in this area. Akyildiz et. al. in [1] presents an excellent survey on wireless mesh network. Introductory studies on Wireless Mesh Networks including its types, applications, implementation, problems and perspectives have been presented in [2],[3],[4]. Work specifically focusing on VoIP systems including the choice of codec and call signaling protocols have been presented in [5],[6],[7]. Authors in [8],[9] present a voice quality measurement tool based on ITU-T E-Model in which quality metrics like R-Factor and Mean Opinion Score have been defined. Analyses conducted in [10] reveal that even single additional VoIP call in the cell could degrade the quality of all the ongoing VoIP calls. In [11],[12],[13], the authors propose various voice quality measures such as delay, jitter, loss rate and R-Score and also propose an Interference capacity model for a wireless mesh network. Several performance optimization schemes like packet Aggregation, Header Compression and Label Based Forwarding have been proposed to enhance the performance of voice in wireless mesh network in [14].

Authors in [15] have performed analysis of Delay and throughput using $\mathrm{M} / \mathrm{D} / 1$ queuing theory by modeling the gateway nodes as independent $\mathrm{M} / \mathrm{D} / 1$ queue stations, and derived closed-form solutions for the bottleneck delay and throughput with linear and grid topologies of wireless mesh network. In [16] authors have analyzed the throughput, packet loss, and delay and also consider the impacts of interference on modeling the mesh hop nodes as $M \backslash M \backslash 1$. Sunny et. al. in [17] have solved the problem of modeling the average delay in an IEEE 802.11 DCF wireless mesh network with a single root node under light aggregate traffic in Wireless Mesh Networks using M\M\1 Queue. In [18], authors have given various delay components to be aggregated to calculate the overall end to end delay for voice traffic. Literature review reveals that $\mathrm{WMN}$ has emerged as a useful technology for various real time applications. This provides motivation for conducting investigation into VoIP performance of WMN.

In this paper, the performance of VoIP over WMN is dependent on number of nodes or mesh routers modeled as linear mesh linear topology. In WMN, the backbone is mainly fixed as the route followed by the traffic is fixed or static. So, under these assumptions, the WMN can be considered as a linear multi-hop network that could be modeled as M/D/1 Queue [19].

\section{MODELLING THE WMN}

Wireless Mesh Network can be modeled using M/D/1 Queue [19]. It is considered that the WMN consists of $\mathrm{n}$ backbone mesh points. The System has been assumed to consist of only one server i.e. the gateway through which the traffic is forwarded onto the Internet. The arrival process has been assumed to be Poisson distributed where as the service time is deterministic. Under such assumptions the system can be modeled by using M/D/1 queue.

This model has been used to find the average delay taken by the VoIP packets from source mesh point to destination mesh point. Each mesh router is a station in the equivalent queuing network representation. The average delay is the expectation of packet delay over all packets. This work has been restricted to intra mesh communication scenarios i.e. cases where mesh clients communicate with each other using the mesh router backbone. 
With Assumption of M/D/1 Queuing theory, the voice packets arrive according to a Poisson process with mean arrival rate $\lambda$, and packets are processed in a deterministic manner with service time s. Suppose that packets are processed in the order they arrive and that $\mathrm{Xi}$ is the service time of the ith arrival. We assume that each random variable $(\mathrm{X} 1, \mathrm{X} 2 \ldots)$ is identically distributed and identical for all voice packets.

\section{Let $s=E[X]=1 / \mu=$ AverageServiceTime

$$
E\left[X^{2}\right]=\text { Second moment of service Time }
$$

According to Pollaczek-Khinchin (P-K) formula:

For a General Distribution,

$$
W=\lambda E\left[X^{2}\right] /(2 *(1-\rho))
$$

Where, $\mathrm{W}$ is the expected wait time in queue and $\rho$ is the utilization factor.

$$
\rho=\lambda / \mu=\lambda E[X]
$$

Applying little's formula to $\mathrm{W}$, we get the expected number of packets in the queue as NQ and the expected number in the system as $\mathrm{N}$ :

$$
\begin{aligned}
& N Q=\lambda W \\
& N Q=\lambda^{2} E\left[X^{2}\right] /(2 *(1-\rho)) \\
& N=\rho+\lambda^{2} E\left[X^{2}\right] /(2 *(1-\rho))
\end{aligned}
$$

When service time is identical for all voice packets i.e. if $M / D / 1$ theory is applied,

$$
E\left[X^{2}\right]=1 / \mu^{2}
$$

Now, the total average packet Queuing delay = Service time +Waiting time in Queue. Therefore, total time taken by VoIP packets in queue and service in a wireless network can be represented as:

$$
\begin{aligned}
& Q_{d}=E[X]+\lambda E\left[X^{2}\right] /(2 *(1-\rho)) \\
& Q_{d}=s+\lambda s^{2} /(2 *(1-\lambda s))
\end{aligned}
$$

where, $Q_{d}$ is the average queuing delay taken by VoIP packets to reach from source client to destination via a mesh router in a network. Now for a Wireless Mesh Network having $\mathrm{n}$ mesh routers, the Eq.(2) can be changed to Eq. (3) by changing $\lambda$ by $n \lambda$. In case of $n$ mesh routers, the net arrival rate will become $n \lambda$.

So, the average queuing delay incurred by VoIP packets from source mesh client to destination (Internet gateway in this case) in a Wireless Mesh Network having $n$ mesh routers [15] can be expressed as equation:

$$
Q_{n d}=s+n \lambda s^{2} /(2 *(1-n \lambda s))
$$

\subsection{Packet Aggregation}

As, we are using G.711 encoder, the default packetization interval for 160 byte payload is $20 \mathrm{~ms}$ i.e. voice packets are transmitted at the rate of $\lambda=50$ packets per second. Taking service time s equal to $.01 \mathrm{sec}$. By increasing the number of mesh points i.e. value of $\mathrm{n}$ in Eq. (3), we get negative value for queuing delay for using more than 1 mesh node. It is due to high value of arrival rate $\lambda$ of voice packets. So, we can decrease this arrival rate by aggregating the packets to a super packet to transmit them at higher packetization interval.

Packet aggregation [14] multiplexes VoIP packets from different connections into one large packet. It aggregates various data packets into a frame to reduce protocol header overhead.

If we consider a VoIP packet of payload 160 bytes emitted once in $20 \mathrm{~ms}$, then we can merge 5 packets into one giving 800 bytes emitted once in $100 \mathrm{~ms}$. With a $100 \mathrm{~ms}$ sample period corresponding to a rate of 10 packets per second, the payload size is $(64000 * 100) /(8 * 1000)=800$ bytes. So, more the value of sample period, lesser will be the arrival rate. Figure 3 show the increase of Analytical Queuing Delay for voice packets with increase in number of mesh points.

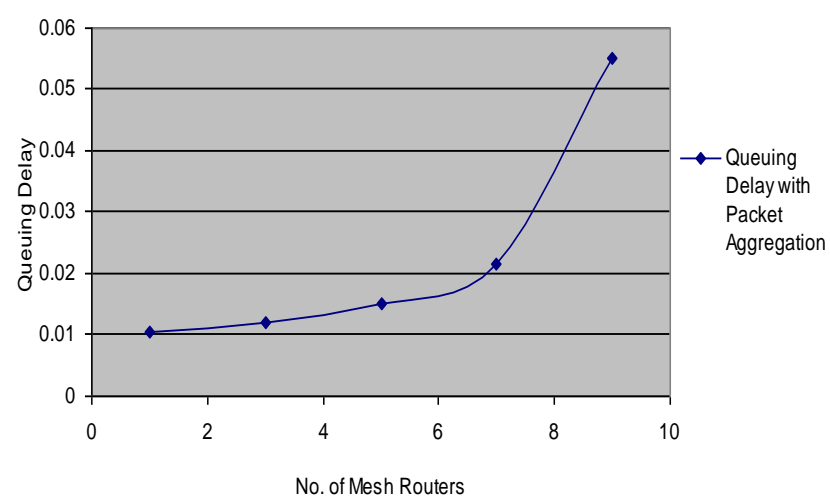

\section{Fig.3. Analytical Average Queuing Delay with varying mesh routers}

Total delay in VoIP applications is comprised of packetization delay, queuing delay, propagation delay, encoding/decoding delay, transmission delay and jitter buffer delay. The end to end delay $\mathrm{D}$ for VoIP traffic in wireless mesh network in one direction from sender to receiver can be represented by the expression [18]:

$$
D=P_{d}+Q_{n d}+J_{d}+\sum_{\substack{\text { Foreachmeshnodei } \\ 1 \leq i \leq n}}\left(P R_{i}+T_{i}\right)
$$

where $P_{d}$ is the packetization delay introduced by encoder G.711 at the source. As, we are using G.711 encoder, the default packetization delay for 800 byte payload will be $100 \mathrm{~ms}$. $\mathrm{Q}_{\mathrm{nd}}$ is the Queuing Delay for mesh network having n mesh nodes which can be calculated from Eq. (3).

$\mathrm{J}_{\mathrm{d}}$ is the Jitter delay incurred by the jitter buffer. In this case, we are taking no jitter buffer delay. $\mathrm{PR}_{\mathrm{i}}$ and $\mathrm{T}_{\mathrm{i}}$ are the propagation and transmission delay incurred at each mesh node $\mathrm{i}$ in the path from the sender to the receiver. Finally, we can aggregate all the delays to find the total average one way delay of VoIP traffic from source mesh client to destination. 


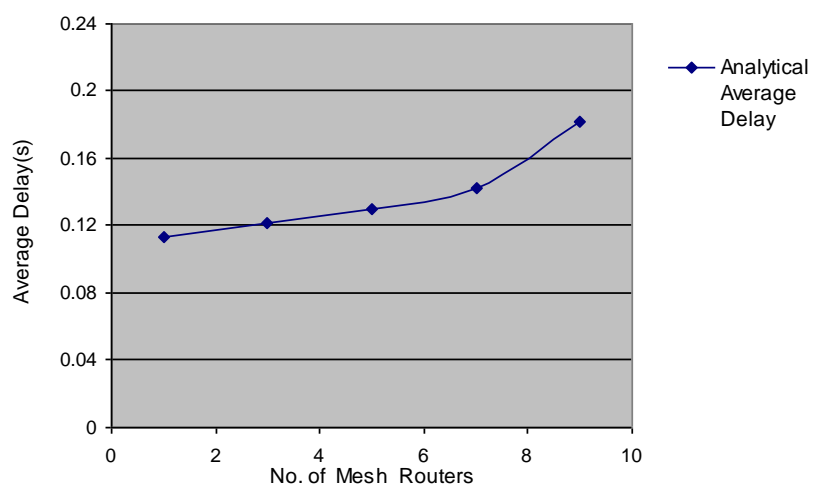

Fig.4. Analytical Average one way Delay from VoIP source to destination with packetization delay of $100 \mathrm{~ms}$

Figure 4 represents increase in Analytical Average one way Delay of voice in Wireless Mesh Network computed using Eq. (4). Voice is passed from source to destination with varying mesh nodes with packetization delay of $100 \mathrm{~ms}$.

\section{SIMULATION AND RESULTS}

To verify the correctness of the model, a WMN scenario has been simulated using Qualnet simulator. Qualnet[20] is a discrete event simulation system having a robust graphical user interface which covers all aspects of the simulation from scenario creation, topology setup, integration of custom protocols, through real-time execution of network models from within the GUI, animation, to post-simulation statistical analysis. Qualnet has been used to simulate high-fidelity models of wireless networks with as many as 50,000 mobile nodes.

Six scenarios of wireless mesh network have been considered in the simulation. The number of mesh nodes or mesh points has been varied in the network to see the influence of the network size on the performance of VoIP traffic in WMN. In the first scenario, the source and destination has been separated by only one mesh access point or hop and then in further scenarios number of mesh access points or hops have been increased to 3, 5, 7, 9 and 11 hops respectively. In each case 5 simulation instances have been run with different seeds. After that average is taken for different cases of a scenario to produce the result.

Figure 5 shows a Scenario Designer Window in Qualnet having five Mesh Routers or Mesh Points numbered 1, 2, 3, 4 and 5 respectively. All nodes are interlinked with each other through wireless subnet and VoIP Traffic is passed between mobile clients numbered with 6 and 7 with interval of $20 \mathrm{~ms}$.

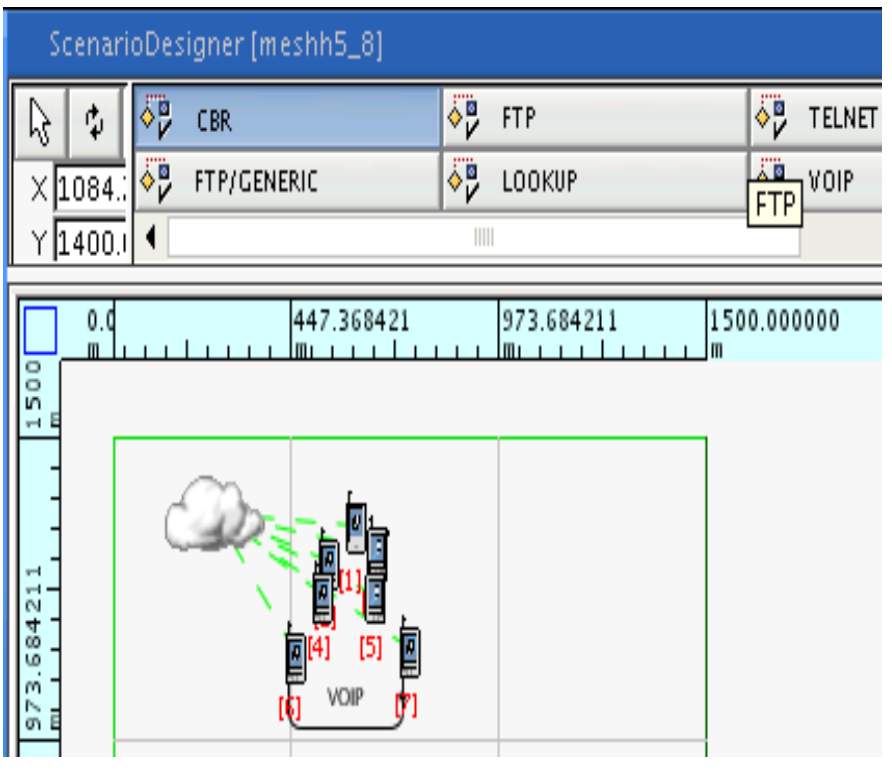

Fig.5. Scenario Designer Window of Qualnet

Figure 6 shows the graph which represents the degradation of voice quality in terms of Mean Opinion Score (MOS) with increase in number of hops (Mesh Routers) in wireless mesh network.

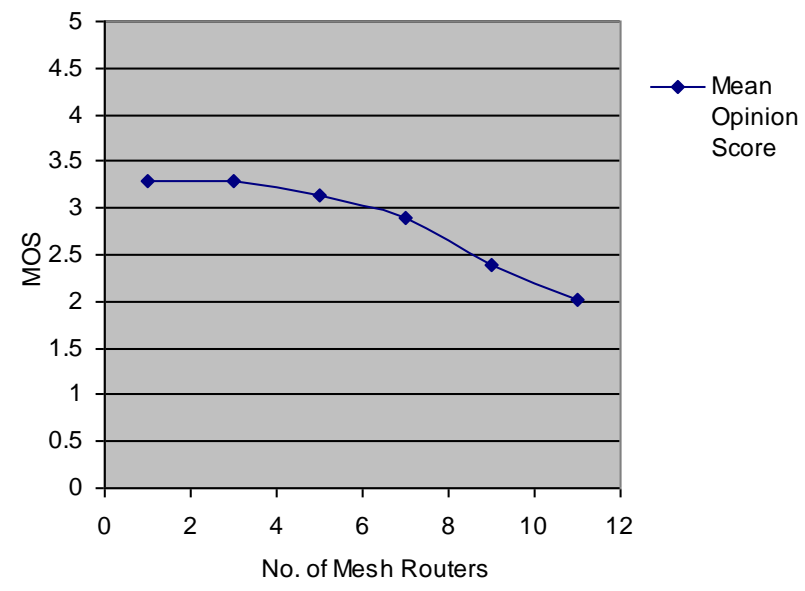

Fig.6. MOS vs No. of Mesh Routers

As we can see in Fig. 6, up to 7 mesh routers voice quality in terms of MOS degrade in almost linear manner but voice quality greatly reduces when more than 7 mesh routers have been introduced in a wireless mesh network. From the graph, it can be seen that voice quality decreases in an exponential manner with increase in number of mesh routers. Fig. 7 shows the degradation of R-Factor with increase in number of hops. 


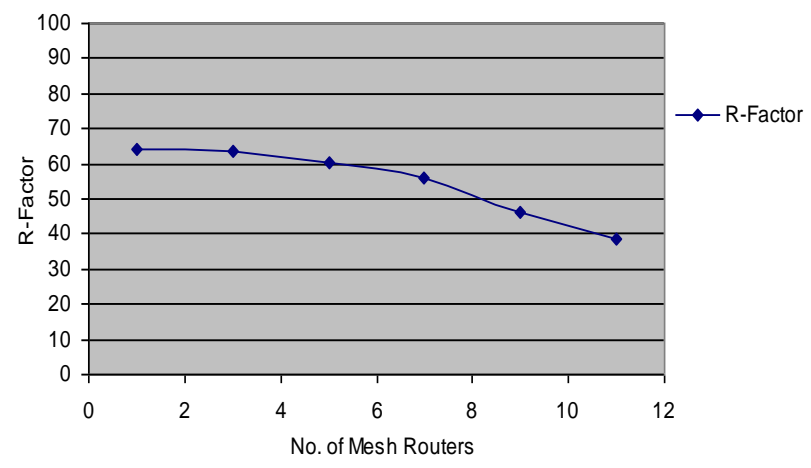

Fig.7. R-Factor vs. No. of Mesh Routers

Fig. 8 shows the increase in Average one way Delay with increase in number of mesh routers in a wireless mesh network when VoIP traffic is transmitted with packetization interval of $20 \mathrm{~ms}$.

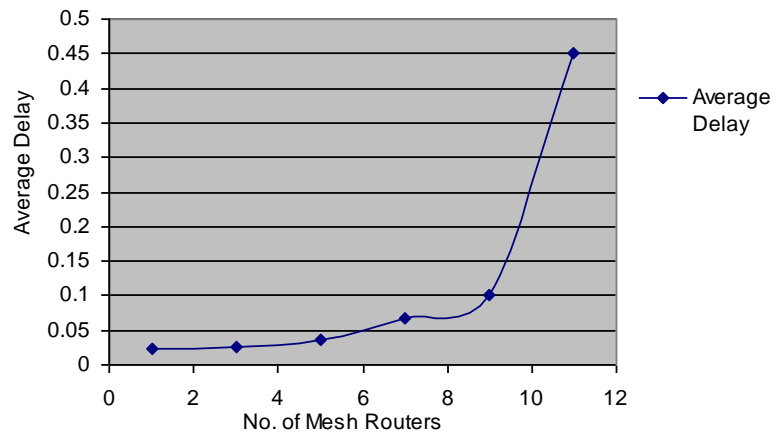

Fig. 8: Average one way delay with varying mesh routers with packetization interval of $20 \mathrm{~ms}$

\section{VALIDATION}

Finally the comparison between the theoretical and simulation results has been presented. For the analytical computation of mean delay from $\mathrm{Eq}(4)$, the arrival rate $\lambda$ is taken as $10 \mathrm{pps}$. The value of $n$ i.e. the number of hops has been varied. Validation tests were run multiple times to determine the accuracy of the analysis. Extensive simulations have been conducted to verify the analytical results.Fig. 9 shows the comparison between Analytical and Simulation Results obtained for average delay incurred by VoIP packets from source to destination with varying number of hops (upto 9) for packetization interval of $100 \mathrm{~ms}$. The simulation results show that the analysis results almost matches the simulation results i.e. both simulation and theoretical delay increases in an exponential manner as the number of mesh hops increases. Thus the $M \backslash D \backslash 1$ model successfully predicts the average delay in wireless mesh networks.

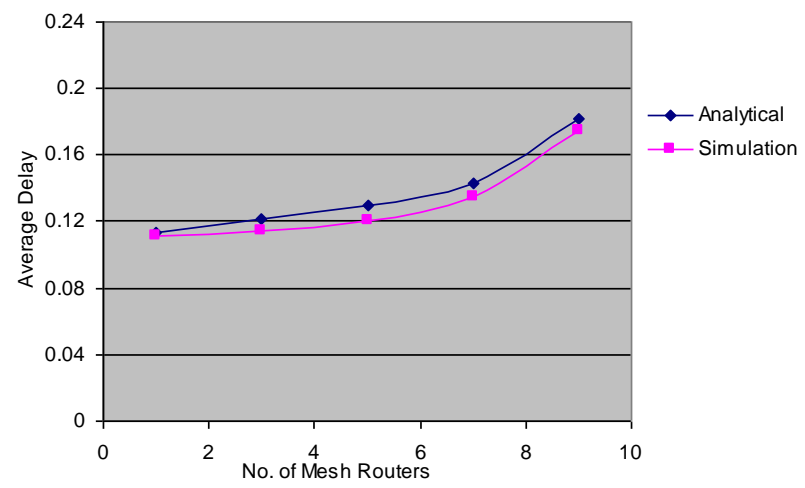

Fig. 9: Comparison of Simulation and Analytical Average Delay with packetization interval of $100 \mathrm{~ms}$.

\section{CONCLUSIONS \& FUTURE WORK}

This paper presents analytical model and simulation results of VoIP traffic over 802.11 based WMN in terms of parameters such as average delay, R-Factor and Mean Opinion Score. Estimation of Average delay in VoIP over WMN with varying number of nodes has been carried out using theoretical analysis and compared with simulation results. The developed model can be added to the existing R-factor for predicting R score when WMN is present in the network. Also the upper limit on number of hops in WMN for carrying the VoIP applications can be estimated. In the future work the scenario involving multi-channel multi-radio WMNs can be explored. Also performance of voice in a WMN can be measured and compared for various proactive and reactive routing protocols. Subsequently, the framework could be extended to model the scenario for non-Poisson arrivals and also for different service distributions.

\section{REFERENCES}

[1] I.F.Akyildiz, X.Wang, "A Survey on Wireless Mesh Networks", IEEE Comm Mag. 2005, pp.23-30, vol 43(9).

[2] R. Bruno, M. Conti, and E. Gregori, "Mesh Networks: Commodity Multihop AdHoc Networks", IEEE Comm. Mag., vol. 37, pp. 123-131, March 2005.

[3] Tzu-Jane and Ju-Wei Chen, "IEEE 802.11 MAC Protocol over Wireless Mesh Networks: Problems and Perspectives", March 2005, 60-63.

[4] Mahmud, S.A., Khan, S., Khan, SH., Al-Raweshidy, H., “A comparison of MANETs and WMNs: commercial feasibility of community wireless networks and MANETs", Proceedings of the 1st international conference on Access networks, 2006, pp: 36:54, 2006.

[5] Schulzrinne, H. and Rosenberg, J., "Internet telephony: architecture and protocols - an IETF perspective", Computer Networks and ISDN Systems, Vol. 31, pp. 237-255, Feb.1999.

[6] Bur Goode, Senior Member, "Voice over Internet Protocol (VoIP)", Proceedings of the IEEE, Vol. 9.

[7] Trad, F. Munir, H. Afifi, "Capacity Evaluation of VoIP in IEEE 802.11e WLAN Environment", IEEE CCNC 2006 Proceedings, September 2002. 
[8] Leandro Carvalho, Edjair Mota, Regeane Aguiar Anderson Barreto, "An E-Model Implementation for Speech Quality Evaluation in VoIP Systems", IEEE Symposium Computers and Communications (ISCC 2005), pp. 1530-1346, IEEE, 2005.

[9] L. Ding and R.A. Goubran, "Speech quality prediction in VoIP using the extended E- model", IEEE GLOBECOM 2003, pp. 3974-3978.

[10] Sachin Garg, Martin Kappes, "Can I add a VoIP call?" Proc. of IEEE ICC'03, vol.2, May 2003.

[11] Xudong Wang, Abhishek Patil and Weilin Wang., "Voip over wireless mesh networks: challenges and approaches", WICON '06: Proceedings of the 2nd annual international workshop on Wireless internet, page 6, New York, USA, 2006.

[12] H.Y. Wei, K. Kim, A. Kashyap and S. Ganguly, "On Admission of VoIP Calls over Wireless Mesh Network", Proceedings of IEEE ICC, 2006.

[13] K. Kyungtae and H. Sangjin, "VoMESH: voice over wireless mesh networks", Proc of IEEE Wireless Communications and Networking Conference, LasVegas, USA, 2006, pp.1938.
[14] S.Ganguly et al., "Performance Optimization for Deploying VoIP Services in Mesh Networks", IEEE Journal on Selected Areas in Communications (JSAC), 2006 pp 2147-2158.

[15] X. Wu, J. Liu, G. Chen, "Analysis of Bottleneck Delay and Throughput in Wireless Mesh Networks", IEEE International Conference on Mobile Ad-hoc and Sensor Systems, 2006.

[16] Yunxia Feng, XingfaShen, Zhigang Gao, Guojun Dai, "Queuing Based Traffic Model for Wireless Mesh Networks", Proc of $15^{\text {th }}$ International Conference on Parallel and Distributed Systems, 2009.

[17] Albert Sunny, Joy Kuri, Saurabh Aggarwal, "Delay modeling for a single-hop wireless mesh network under light aggregate raffic," online version available at http://www.arxiv.org/abs/1009.0448

[18] M. Karam and F. Tobagi, "Analysis of the Delay and Delay Jitter of Voice Traffic in the Internet", Computer Network Magazine, vol 40, no 6, December 2002, pp. 711726.

[19] Queuing web link available: - http://en.wikipedia.org/ wiki/M/D/1_model

[20] Scalable Network Technologies, Inc. "QualNet User's Manual, version 3.6.", http://www.scalable-networks.com/ 2003. 